\title{
Reversibility of retinal ischemia due to central retinal artery occlusion by hyperbaric oxygen
}

\author{
This article was published in the following Dove Press journal: \\ Clinical Ophthalmology \\ 29 December 2016 \\ Number of times this article has been viewed
}

\author{
Amir Hadanny ${ }^{1,2}$ \\ Amit Maliar' \\ Gregory Fishlev' \\ Yair Bechor' \\ Jacob Bergan' \\ Mony Friedman' \\ Isaac Avni ${ }^{2,3}$ \\ Shai Efrati ${ }^{1,2,4,5}$ \\ 'Sagol Center for Hyperbaric Medicine \\ and Research, Assaf Harofeh Medical \\ Center, Zerifin, Israel; ${ }^{2}$ Sackler School \\ of Medicine, Tel-Aviv University, \\ Tel-Aviv, Israel; ${ }^{3}$ Opthalmology \\ Department, Assaf Harofeh Medical \\ Center, Zeirifin, Israel; ${ }^{4}$ Research and \\ Development Unit, Assaf Harofeh \\ Medical Center, Zerifin, Israel; ${ }^{5}$ Sagol \\ School of Neuroscience, Tel-Aviv \\ University, Tel-Aviv, Israel
}

Correspondence: Amir Hadanny Sagol Center for Hyperbaric Medicine and Research, Assaf Harofeh Medical Center, Zerifin 70300 , Israel

Tel +972 54470 738I

Fax +972 89779748

Email amir.had@gmail.com
Purpose: Ischemic retinal damage can be reversed by hyperbaric oxygen therapy (HBOT) as long as irreversible infarction damage has not developed. However, the time window till irreversible damage develops is still unknown. The study aim was to evaluate the effect of HBOT and determine possible markers for irreversible retinal damage.

Materials and methods: Retrospective analysis of 225 patients treated with HBOT for central retinal artery occlusion (CRAO) in 1999-2015. One hundred and twenty-eight patients fulfilled inclusion/exclusion criteria: age $>18$ years, symptoms $<20$ hours, and best-corrected visual acuity (BCVA) $<0.5 \log$ MAR.

Results: Time delay from symptoms to treatment was $7.8 \pm 3.8$ hours. The BCVA was significantly improved after HBOT, from $2.14 \pm 0.50$ to $1.61 \pm 0.78(P<0.0001)$. The proportion of patients with clinically meaningful visual improvement was significantly higher in patients without cherry-red spot (CRS) compared to patients with CRS at presentation ( $86.0 \%$ vs $57.6 \%$, $P<0.0001)$. The percentage of patients with final BCVA better than 1.0 was also significantly higher in patients without CRS vs patients with CRS at presentation $(61.0 \%$ vs $7.1 \%, P<0.0001)$. There was no correlation between CRS and the time from symptoms. HBOT was found to be safe, and only $5.5 \%$ of patients had minor, reversible, adverse events.

Conclusion: HBOT is an effective treatment for non-arteritic CRAO as long as CRS has not formed. The fundus findings, rather than the time delay, should be used as a marker for irreversible damage.

Keywords: HBOT, hyperbaric oxygen, central retinal artery occlusion, cherry-red spot, CRAO, retinal ischemia

\section{Introduction}

Central retinal artery occlusion (CRAO) is a serious, relatively common, ophthalmologic condition with a poor prognosis. The incidence of acute CRAO is estimated at 8.5 in 100,000 people. ${ }^{1}$ The natural history of the disease is devastating, with $92 \%$ of patients left with poor visual acuity of counting fingers or less, and only $8 \%$ may experience improvement. $^{2-4}$

The retina is the organ that has the highest oxygen consumption rate per size in the human body, utilizing $13 \mathrm{~mL} / 100 \mathrm{~g} / \mathrm{min}$, and is therefore very sensitive to ischemia. Animal studies have shown total retinal ischemia, or anoxia, can be fully reversed if the retina is reoxygenized within 97 minutes of onset. ${ }^{5}$ As opposed to animal models, in humans, there is no clear timeline until irreversible anoxic retinal damage occurs. Some authors suggest elapsed time of around 6-6.5 hours ${ }^{6}$ but due to the large variability between patients, types of occlusion, and residual perfusion, this time frame is not reliable and thus another biologically based marker is needed. 
Despite the optimism generated by anecdotal reports and uncontrolled studies, none of the treatments aiming to target CRAO have been proven to be safe and effective. ${ }^{7}$ Conventional treatment regimens aimed at promoting downstream movement of the embolus by lowering intraocular pressure (IOP) and producing vasodilatation have been largely unsuccessful. ${ }^{8-10}$ The use of local intra-arterial fibrinolysis (LIF) has been evaluated in a multicenter, prospective, randomized clinical trial (the European Assessment Group for Lysis in the Eye, or EAGLE, study) in 82 patients with non-arteritic CRAO within 20 hours of symptom onset. ${ }^{11}$ Unfortunately, LIF had similar efficacy as conservative standard treatment (CST) with significantly higher rate of adverse reactions $(37 \%){ }^{11}$

Hyperbaric oxygen therapy (HBOT) includes the inhalation of $100 \%$ oxygen at pressures exceeding 1 atmosphere absolute (ATA) used to enhance the amount of oxygen dissolved in the body tissues. During HBOT treatment, the arterial $\mathrm{O}_{2}$ tension typically exceeds $2,000 \mathrm{mmHg} .{ }^{12}$ The proposed role for hyperbaric oxygen in CRAO is to increase the oxygen delivery to the ischemic tissue until spontaneous or assisted reperfusion occurs. CRAO is a US Food and Drug Administration approved indication for HBOT, with the highest level of evidence when administrated within the first 8-12 hours from the onset of the visual loss. ${ }^{13-20}$ It should be noted that most studies were based on a relatively small sample size without proper exclusion criteria, such as arteritic CRAO or cilioretinal artery sparing. There is a significant difference between patients in relation to residual retinal blood flow and that may be the reason for the large variability in visual outcomes, irrespective of the time delays until treatment. ${ }^{2}$ Based on the current available data, it is difficult to predict which patients will respond to HBOT beyond the recognition that the earlier the better (minimizing the retinal ischemic time, maximizing the potential for visual recovery). ${ }^{21}$

The primary objective of this study was to evaluate the effect of HBOT and determine possible markers for irreversible retinal damage. The study includes the largest cohort published to date, of non-arteritic CRAO patients with no cilioretinal artery sparing.

\section{Materials and methods Study design}

From January 1999 to December 2015, 232 patients with suspected CRAO were treated with hyperbaric oxygen for retinal artery occlusion in Sagol Center for Hyperbaric Medicine and Research at Asaf Harofeh Medical Center, Tel-Aviv, Israel. Visual acuity and clinical evaluations of all patients were performed in the ophthalmology department of Asaf Harofeh Medical Center.

The data were collected retrospectively from medical records and included age, sex, medical conditions, chronic medications, ophthalmologic history, time delay from symptoms to treatment, funduscopic findings, IOP, HBOT protocol and number of sessions, adverse events, and visual acuity outcome. Diagnosis was based on clinical symptoms and fundus examination.

Inclusion criteria were: age older than 18 years, nonarteritic CRAO with symptoms lasting for less than 20 hours, and best-corrected visual acuity (BCVA) worse than 0.5 logarithm of the minimum angle of resolution ( $\log M A R)$, which is worse than the Snellen equivalent of 20/63 (determined with the use of an Early Treatment Diabetic Retinopathy Study [ETDRS] chart). Exclusion criteria were: patent cilioretinal artery, no documented BCVA, BCVA $<0.5$ $\log$ MAR, arteritic CRAO, other final diagnosis, iatrogenic CRAO, and branch retinal artery occlusion (BRAO).

Assaf HaRofeh Medical Center Institutional Review Board approval was obtained for retrospective analysis of all cases used in this study, as well as waived patient consent.

\section{Hyperbaric oxygen protocol}

Treatment was given in 2-2.4 ATA, 100\% oxygen, 90-minute sessions, three times in the first 24 hours and once daily thereafter. Treatment was discontinued when no further improvement in BCVA was observed in two consecutive treatments. All HBOT sessions were performed in a multiplace hyperbaric chamber (Starmed 2700, HAUX-Life Support- GmbH, Germany).

\section{Other treatments}

Either an ocular massage, anterior chamber paracentesis, oral aspirin, oral acetazolamide, or topical beta-blocker was applied prior to HBOT according to a certified ophthalmologist's decision, according to IOP.

\section{Origin of CRAO investigation}

Detailed neurologic and cardiologic examinations were performed during hospitalization to establish the cause of the occlusion. Clinical examination, echocardiography, and duplex ultrasound of the carotid arteries were performed as part of the investigation.

\section{Outcomes}

The primary efficacy end point was change in the BCVA at discharge after therapy compared to the BCVA before therapy. This was measured by the difference from baseline 
$\log$ MAR at the discharge exam. The logMAR was assessed with the ETDRS charts in a standardized protocol. A clinically significant visual improvement was defined as a decrease in $\log$ MAR of $0.3 .{ }^{22,23}$ The numeric $\log$ MAR values for profound low vision categories (ie, counting fingers, hand movement, light perception, and no light perception) were used as proposed by Lange et al. ${ }^{23}$

The safety of HBOT in terms of adverse reactions was monitored and analyzed.

\section{Statistics}

Data are expressed as mean \pm standard deviation (SD) for parametric variables and frequencies and percentages for nonparametric variables. BCVA and other numeric variables analyses were performed using pairwise/independent Student's $t$-test. Other non-parametric covariates were analyzed using the chisquare or Fisher's exact test (where appropriate) to identify significant variables $(P<0.05)$. Normality of parametric data was performed using the Kolmogorov-Smirnov test.

Multiple linear regression model and multivariate logistic regression models were performed to control for potential confounders and to determine independent predictors for clinical outcome. Covariates included in the models were age, sex, chronic medical conditions (diabetes mellitus, hypertension, hypercholesterolemia, ischemic heart disease, other vasculopathy, renal disease, previous stroke), active smoking, chronic medications (anti-aggregation, anti-coagulation, statins), involved eye, time delay from symptoms to diagnosis, fundus findings at presentation (cherry-red spot [CRS], plaques, boxcarring, IOP), other treatments (aspirin, paracentesis, eye massage, acetazolamide, eye topical drugs), total number of HBOT sessions, and BCVA at presentation.

In these models, highly intercorrelated independent variables $(r>0.7)$ were avoided. Odds ratios (ORs) and 95\% confidence intervals (95\% CIs) were calculated. Methods were performed using the Statistical Package for the Social Sciences (SPSS) v.21 software.

\section{Results}

Of the 232 patients treated at Sagol Center for Hyperbaric Medicine and Research between January 1999 and December 2015, 128 fulfilled the inclusion criteria and were included in the final analysis (Figure 1). Of the excluded patients, seven were misdiagnosed as CRAO, 53 had other etiology of retinal artery occlusion (branch, arteritic, or iatrogenic), 14 had patent cilioretinal artery, 14 lacked documentation of BCVA at baseline or after HBOT, 14 were treated later than 20 hours from symptoms, and another two had visual acuity at presentation better than $0.5 \log$ MAR.

\section{Patients' characteristics}

Patients' characteristics are detailed in Table 1. The mean age was $66.4 \pm 13.1$ years and $69 \%$ were males. Hypertension and hypercholesterolemia were the most prevalent chronic medical conditions ( $63.3 \%$ and $44.5 \%$, respectively). Half of the patients were treated with anti-aggregation $(43 \%)$ or anti-coagulation (8.6\%) drugs prior to the CRAO event.

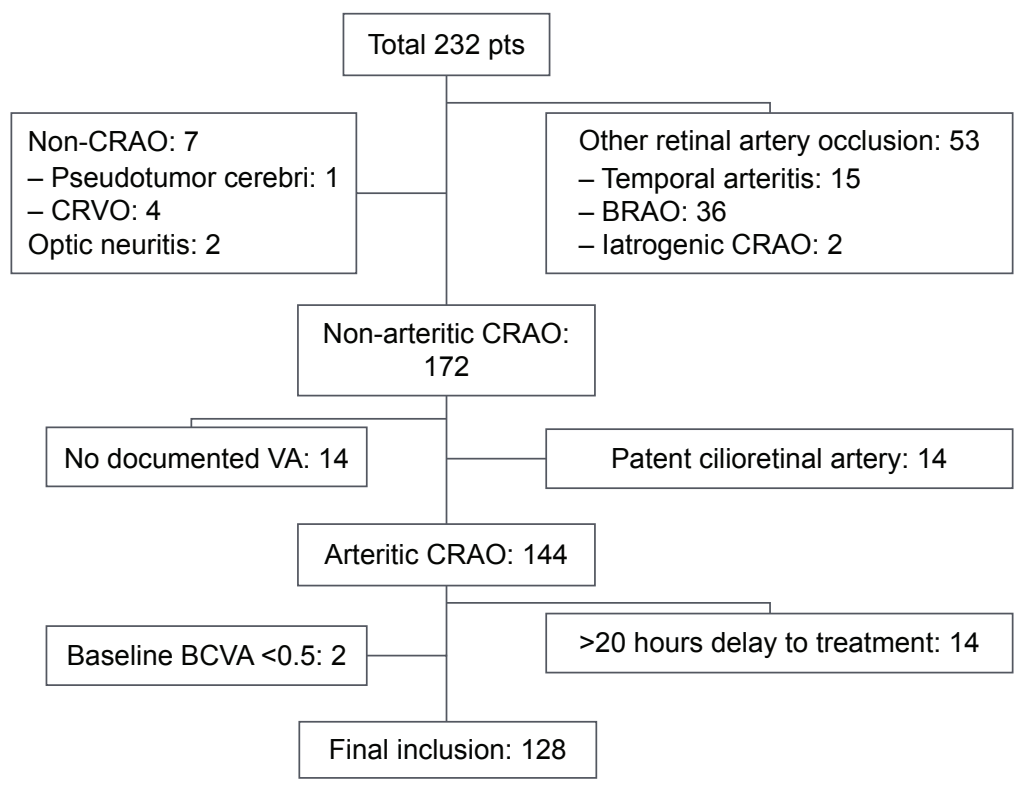

Figure I Patients flow.

Abbreviations: Pts, patients; CRAO, central retinal artery occlusion; CRVO, central retinal vein occlusion; BRAO, branch retinal artery occlusion; VA, visual acuity; BCVA, best-corrected visual acuity. 
Table I Patients baseline characteristics, symptoms and treatments

\begin{tabular}{|c|c|c|c|c|}
\hline Characteristic & $\begin{array}{l}\text { НВОТ } \\
(n=128)\end{array}$ & $\begin{array}{l}\text { CRS } \\
(n=85)\end{array}$ & $\begin{array}{l}\text { Non-CRS } \\
(n=43)\end{array}$ & Significance \\
\hline Males & 88 (68.8\%) & $6 I(7 I .8 \%)$ & 27 (62.8\%) & $P=0.30 \mathrm{I}$ \\
\hline Age, years & $66.4 \pm 13.1$ & $67.1 \pm 12.2$ & $65.0 \pm 14.8$ & $P=0.383$ \\
\hline \multicolumn{5}{|l|}{ Chronic medical conditions } \\
\hline Diabetes mellitus II & 27 (2I.1\%) & $18(2 \mid .2 \%)$ & $9(20.9 \%)$ & $P=0.974$ \\
\hline Hypertension & $8 \mathrm{l}(63.3 \%)$ & $53(62.4 \%)$ & $28(65.1 \%)$ & $P=0.759$ \\
\hline Hypercholesterolemia & 57 (44.5\%) & $36(42.4 \%)$ & $2 \mathrm{I}(48.8 \%)$ & $P=0.486$ \\
\hline Active smoking & 43 (33.6\%) & $30(35.3 \%)$ & $13(30.2 \%)$ & $P=0.567$ \\
\hline Ischemic heart disease & $38(29.7 \%)$ & $26(30.6 \%)$ & 12 (27.9\%) & $P=0.754$ \\
\hline Stroke & 12 (9.4\%) & $8(9.4 \%)$ & $4(9.3 \%)$ & $P=0.984$ \\
\hline Other vasculopathy & $22(17.2 \%)$ & $16(8.8 \%)$ & $6(14.0 \%)$ & $P=0.490$ \\
\hline \multicolumn{5}{|l|}{ Chronic medications } \\
\hline Anti-aggregations & 55 (43.0\%) & $37(43.5)$ & I8 (4I.9\%) & $P=0.857$ \\
\hline Anti-coagulation & II (8.6\%) & 10 (II.8\%) & I (2.3\%) & $P=0.1$ \\
\hline Statins & $42(32.8 \%)$ & 27 (3I.8\%) & 15 (34.9\%) & $P=0.723$ \\
\hline \multicolumn{5}{|l|}{ Involved eye } \\
\hline Right & $55(43 \%)$ & $38(44.7 \%)$ & 17 (39.5\%) & $P=0.577$ \\
\hline Left & $73(57 \%)$ & $47(55.3 \%)$ & $26(60.5 \%)$ & \\
\hline Time from symptoms to treatment & $7.8 \pm 3.8(I-20)$ & $8.1 \pm 3.7(3-19)$ & $6.8 \pm 3.7(1-20)$ & $P=0.06$ \\
\hline Wakeup with symptoms & $13(10.2 \%)$ & $10(11.8 \%)$ & $3(7 \%)$ & $P=0.447$ \\
\hline Intraocular pressure & $13.1 \pm 4.6$ & $12.2 \pm 3.2$ & $14.9 \pm 6.3$ & $P=0.013$ \\
\hline \multicolumn{5}{|l|}{ Fundus findings } \\
\hline Plaque & $20(15.6 \%)$ & $13(15.3 \%)$ & $7(16.3 \%)$ & $P=0.885$ \\
\hline CRS & 85 (66.4\%) & - & - & - \\
\hline Boxcarring & $27(21.1 \%)$ & $20(23.5 \%)$ & 7 (16.3\%) & $P=0.342$ \\
\hline \multicolumn{5}{|l|}{ Other treatment } \\
\hline Aspirin & $10(7.8 \%)$ & $6(7.1 \%)$ & $4(9.3 \%)$ & $P=0.655$ \\
\hline Paracentesis & 59 (46.1\%) & 47 (55.3\%) & $12(27.9 \%)$ & $P=0.003$ \\
\hline Massage & $36(28.1 \%)$ & $23(27.1 \%)$ & $13(30.2 \%)$ & $P=0.706$ \\
\hline PO acetazolamide & $28(21.9 \%)$ & $22(25.9 \%)$ & $6(14 \%)$ & $P=0.123$ \\
\hline Eye acetazolamide & $20(15.6 \%)$ & $14(16.5 \%)$ & $6(14 \%)$ & $P=0.7 \mathrm{II}$ \\
\hline Brimonidine & $9(7.0 \%)$ & $5(5.9 \%)$ & $4(9.3 \%)$ & $P=0.475$ \\
\hline \multicolumn{5}{|l|}{ Number of HBOT sessions } \\
\hline Median: 4 & $4.0 \pm 1.2$ & $3.9 \pm 1.2$ & $4.1 \pm 1$ & $P=0.456$ \\
\hline
\end{tabular}

Notes: Most patients were males with an average age of 66.4 years. The most prevalent diseases were hypertension and hypercholesterolemia. Interestingly, $43 \%$ were taking aspirin daily prior to CRAO. Two-thirds of the patients had CRS at presentation. Compared to patients with CRS, patients without CRS at presentation had comparable time delays to treatment yet with higher mean IOP. Both patients' groups received similar number of HBOT sessions. Bold text marks statistical significance $(P<0.05)$.

Abbreviations: HBOT, hyperbaric oxygen therapy; CRS, cherry-red spot; PO, by mouth; CRAO, central retinal artery occlusion; IOP, intraocular pressure.

In 85 patients $(66.4 \%)$, the origin of occlusion remained unknown after the investigation at presentation. Twenty-three patients (18\%) had carotid atherosclerosis disease and in 14 patients (11\%) the origin of the emboli was from heart.

The mean time delay from symptoms to treatment was $7.8 \pm 3.8$ hours. The average number of HBOT sessions was $4.0 \pm 1.2$.

\section{Presentation and funduscopic findings}

The right eye was affected in $43 \%$ of the cases. The IOP was $13.1 \pm 4.6 \mathrm{mmHg}$. Two-thirds $(66.4 \%)$ of the patients had CRS at presentation.

\section{Visual outcome}

The BCVA (logMAR) after HBOT compared with the baseline showed a significant mean improvement of $0.526 \pm 0.688$, from $2.14 \pm 0.50$ to $1.61 \pm 0.78(P<0.0001)$.

The proportion of patients with clinically significant visual improvement (change in $\log M A R \geq 0.3$ ) was $67.2 \%$. The percentage of those with final BCVA better than 1.0 $\log$ MAR was $25.0 \%$.

\section{Visual outcome as function of baseline fundus findings}

Patients without CRS at presentation had a significantly larger BCVA improvement than patients with observed 
A

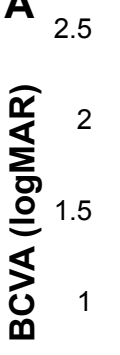

0.5

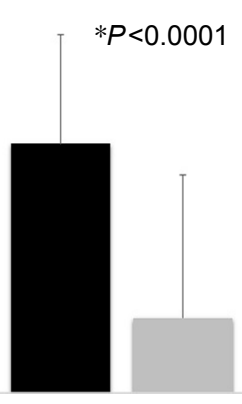

Non-CRS

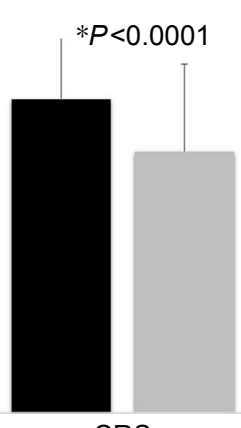

CRS
B
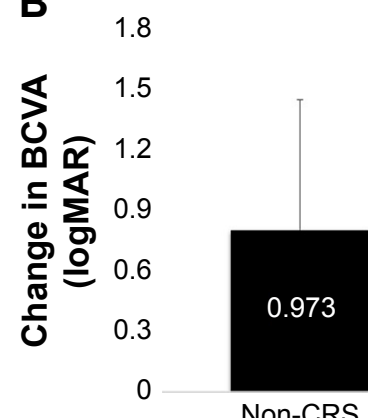

$* P<0.0001$

Non-CRS

Baseline Post-HBOT

Figure 2 HBOT effect on BCVA in patients with and without CRS.

Notes: (A) Shows significant improvements in BCVA after HBOT compared to baseline in both groups. (B) Shows that the mean change in BCVA was significantly larger in the non-CRS group. $*_{\text {indicates }} P<0.05$.

Abbreviations: CRS, cherry-red spot; HBOT, hyperbaric oxygen therapy; BCVA, best-corrected visual acuity.

CRS $(0.973 \pm 0.782$ vs $0.300 \pm 0.506, P<0.0001)$ (Figure 2). The proportion of patients with clinically significant visual improvement was significantly larger in patients without CRS compared to patients with CRS at presentation $(86.0 \%$ vs $49.4 \%, P<0.0001)$. The percentage of patients with final BCVA better than 1.0 logMAR was significantly higher in patients without $\mathrm{CRS}$ than patients with $\mathrm{CRS}$ at presentation $(61.0 \%$ vs $7.1 \%, P<0.0001)$ (Table 2$)$. The visual outcome of additional 14 patients with time delay from symptoms onset to treatment over 20 hours is shown in Table S1.

Notably, there was an almost significant trend toward a difference in the time delay from symptoms to treatment between patients with CRS (mean 8.1 \pm 3.7 hours, range 3-19) and without CRS (mean 6.8 \pm 3.7 hours, range $1-20)(P=0.06)$.

Presence of plaque ( $P=0.710$ ) or boxcarring (cattle trucking) $(P=0.968)$ in fundus examination at presentation did not have any effect on visual outcome.

\section{Visual outcome predictors}

Forward stepwise multivariate linear regression model in 118 patients with complete data found time delay from symptoms to treatment, CRS at presentation, and baseline BCVA statistically significant predictors for visual outcome.

Age, sex, anti-aggregation and anti-coagulation drugs, chronic medical conditions, IOP, other funduscopic findings, paracentesis and eye massage, and number of HBOT sessions had no effect on the change in BCVA.

The finding of CRS at presentation had a linear estimate of -0.787 (95\% CI [0.555-1.018], $P<0.0001$ ), that is, presence of CRS decreases the gain in logMAR after treatment by 0.787 (Figure S1).

Time delay from symptoms to treatment had a linear estimate of -0.030 ( $95 \%$ CI [0.04-0.057], $P=0.026$ ), that is, for each hour delay, the gain in $\log M A R$ after treatment will decrease by 0.03 (0.022 in the EAGLE study $\left.{ }^{11}\right)$.

Forward stepwise logistic regression model shows an observed CRS at presentation and length of time delay from symptoms to treatment increase the risk for non-clinically significant improvement (change in $\log$ MAR $<0.3$ ) by 13.51 ([2.964-58.345], $P=0.001)$ and 1.25 ([1.099-1.422], $P=0.001$ ), respectively (Figure $\mathrm{S} 1$ ).

Table 2 Analysis of BCVA

\begin{tabular}{|c|c|c|c|c|}
\hline Outcome & $\begin{array}{l}\text { All patients } \\
(n=128)\end{array}$ & $\begin{array}{l}\text { CRS } \\
(n=85)\end{array}$ & $\begin{array}{l}\text { Non-CRS } \\
(n=43)\end{array}$ & Significance \\
\hline Baseline logMAR & $2.14 \pm 0.50$ & $2.26 \pm 0.38$ & $1.90 \pm 0.61$ & $P=0.001$ \\
\hline Discharge logMAR & $1.62 \pm 0.78$ & $1.96 \pm 0.49$ & $0.92 \pm 0.80$ & $P<0.000$ I \\
\hline Change in logMAR & $0.526 \pm 0.688$ & $0.300 \pm 0.5 I$ & $0.973 \pm 0.78$ & $P<0.0001$ \\
\hline $\begin{array}{l}\text { Clinically significant visual } \\
\text { improvement } \geq 0.3 \text { logMAR }\end{array}$ & $86(67.2 \%)$ & 49 (57.6\%) & 37 (86\%) & $P=0.001$ \\
\hline $\log M A R \leq 1$ & $32(25.0 \%)$ & $6(7.1 \%)$ & $26(60.5 \%)$ & $P<0.000$ I \\
\hline
\end{tabular}

Notes: After HBOT, two-thirds of the patients had clinically significant improvement and a quarter had BCVA of I or less at discharge. Patients without CRS at presentation had significantly larger improvements than those with CRS. Bold text marks statistical significance $(P<0.05)$.

Abbreviations: BCVA, best-corrected visual acuity; CRS, cherry-red spot; logMAR, logarithm of the minimum angle of resolution; HBOT, hyperbaric oxygen therapy. 
Age, sex, anti-aggregation and anti-coagulation drugs, chronic medical conditions, IOP, other funduscopic findings, other treatments applied (oral aspirin, paracentesis, eye massage, oral acetazolamide, different eye topical drugs), and number of HBOT sessions had no significant effect on the visual change (Table S2).

Forward stepwise logistic regression model found presence of CRS at presentation and baseline BCVA increase the risk for bad visual outcome (discharge logMAR $>1$ ) by 16.488 ([4.857-55.979], $P<0.0001)$ and 3.993 ([1.277-12.490], $P=0.017$ ), respectively (Table S3). Time delay from symptoms to treatment, age, sex, anti-aggregation and anticoagulation drugs, chronic medical conditions, IOP, other funduscopic findings, other treatments applied (oral aspirin, paracentesis, eye massage, oral acetazolamide, different eye topical drugs), and number of HBOT sessions were not statistically significant in this model.

\section{Safety}

Seven patients in total experienced minor adverse events (5.5\%): three patients (2.3\%) experienced mild barotraumas which were fully recovered within $2-3$ days; two patients $(1.5 \%)$ had otalgia without barotrauma signs and one of them $(0.7 \%)$ underwent myringotomy in order to continue HBOT sessions; one patient $(0.7 \%)$ suffered from mild epistaxis unrelated to barotrauma which stopped spontaneously; one patient $(0.7 \%)$ experienced dyspnea during one of the sessions and recovered minutes after the session.

As compared to the HBOT intervention, in the EAGLE study, $2 \%$ of the CST group and $4.5 \%$ of the LIF group experienced serious adverse events. Minor adverse events were experienced by $2 \%$ and $34 \%$ of the CST and LIF groups, respectively. ${ }^{11}$

\section{Discussion}

Previous case and cohort studies have shown efficacy using HBOT for patients with retinal artery occlusion in different time frames. ${ }^{13-20,24}$ However, some of the cases were BRAO, arteritic retinal occlusion, or CRAO with patent cilioretinal arteries. The present study evaluated the therapeutic effect of HBOT in the largest cohort published so far of patients suffering from acute non-arteritic CRAO without patent cilioretinal artery. The most important predictor in the current study for the success of the treatment was no CRS at fundus examination. The improvement in patients without CRS was prominent, with $86 \%$ gaining clinical improvement (logMAR change $\geq 0.3$ ) and over $60 \%$ gaining BCVA $\leq 1$ $\log$ MAR. Based on this finding, it can be concluded that CRS can serve as a marker for irreversible anoxic retinal damage to be used for patients candidates for HBOT, as well as in future studies designed to evaluate different interventions aiming to reverse retinal ischemia.

\section{Retinal dual blood supply}

The retina has a dual blood supply system: cells whose nuclei occupy the vascularized inner retina (including ganglion, bipolar, and Muller glial cells) receive their oxygen from the central retinal artery, whereas the photoreceptors and retinal pigment epithelium, located within the capillary-free outer retina, are supplied by the adjacent choroid that forms part of the posterior ciliary arterial circulation..$^{25,26}$

In CRAO, the pan-retinal non-perfusion causes inner retinal layers (ganglion cell layer and inner nuclear layer) ischemia represented by diffuse inner retinal whitening within a few minutes, increasing in severity over the next few hours. The macular region is the most susceptible for ischemia, therefore opacification is usually confined to the posterior pole; whereas, in more peripheral locations, the nonperfused inner retina remains transparent. The opacification in the macula is absent from the foveola since it contains only the photoreceptor layer, nourished by the choroidal circulation. The contrast between peri-foveolar whitening due to infarction and the retinal pigment epithelium under the foveola generates the classic CRS finding (Figure 3). ${ }^{27}$

\section{CRS as a sign of infarction}

Recently, optical coherence tomography (OCT) confirmed that this opacification and swelling of the posterior pole, sparing the foveola (CRS), represent an ischemic infarction of the inner retinal tissues. ${ }^{28}$ When the inner retinal tissues suffer an irreversible infarct, their optical density increases and shadows over the lower optical intensity of the outer retina. ${ }^{28}$

Animal models of ophthalmic artery occlusion have shown that, as in cerebral ischemia, acute circulatory arrest leads to adenosine triphosphate (ATP) depletion and ion pumps failure which is reversible only within the first 97 minutes. ${ }^{5,29}$ Severe irreversible inner retinal changes and tissue infarction quickly follow as neurons progress along the oncotic cell-death pathway between 97 and 280 minutes from the onset. ${ }^{5}$ However, even in the animal models, there is a marked interindividual variation in the relationship between the time of occlusion and irreversible retinal damage. Animals with known atherosclerosis tolerated longer periods of ischemia compared to healthy subjects. ${ }^{5}$ Humans present even larger variability due to patients' previous medical history, type of occlusion, and residual perfusion. Thus, the time to irreversible changes and infarct in humans is unknown. The results of this study further emphasize the wide variability in 

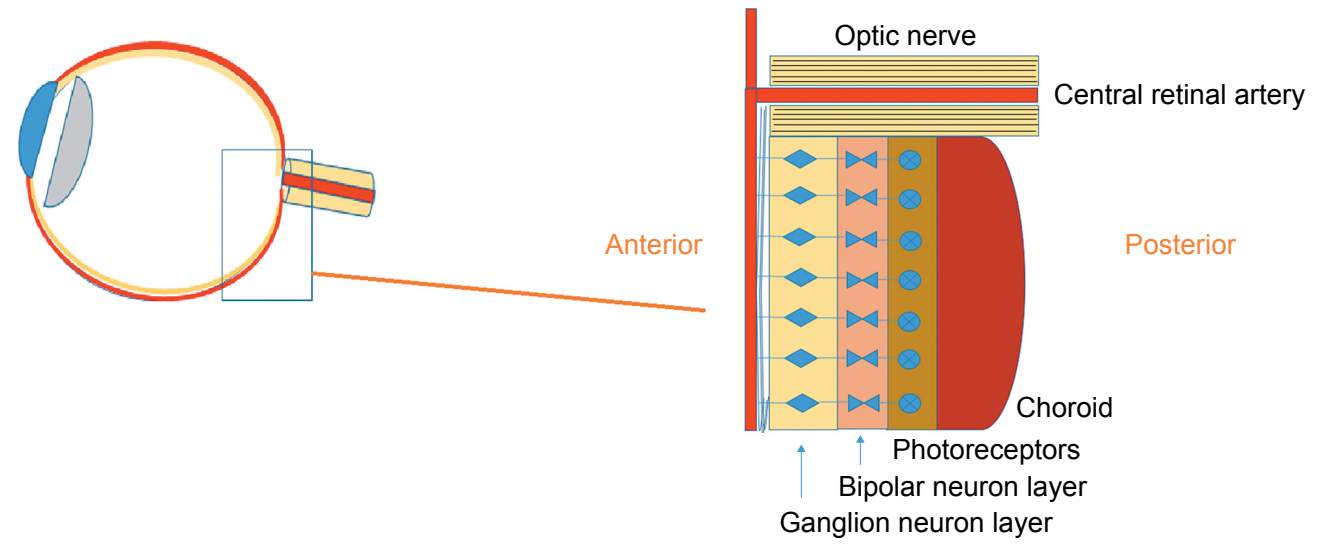

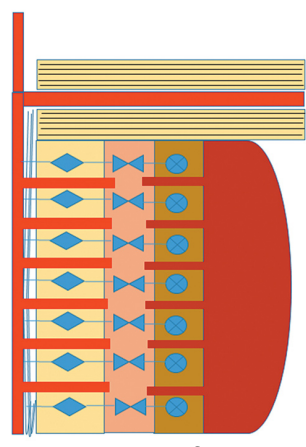

Normal perfusion

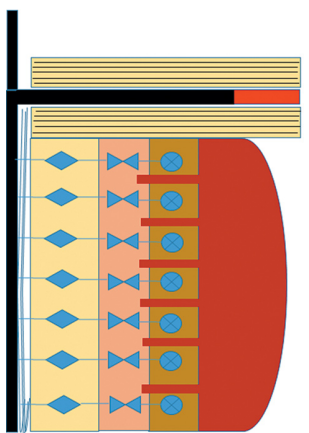

Ischemia (Non-CRS)

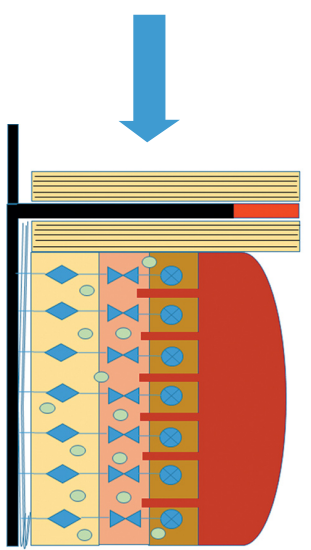

Effective HBOT

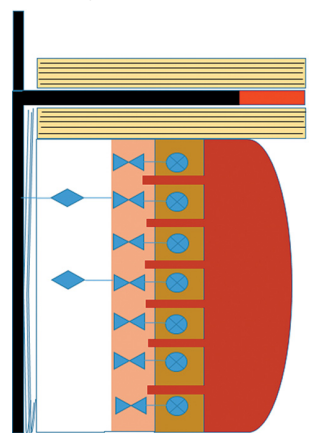

Infarction (CRS)

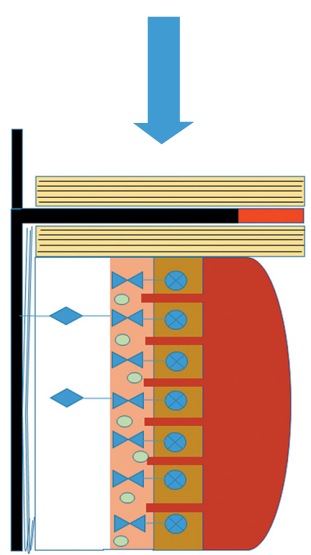

Non-effective HBOT

Figure 3 The ischemic cascade in the retina. At normal perfusion, the retina has a double circulation from both choroidal and retinal systems. When the central retinal artery is occluded, the inner retina suffers ischemia. Depending on the residual blood flow from the choroidal system, with time the ischemia can result in anoxia of the inner retina. The anoxia can be clinically seen as a CRS since when the inner retina is infarcted there are opacification changes. HBOT can effectively increase oxygen diffusion from the patent choroidal system to the inner retina and reverse the ischemic damage as long as irreversible infarction has not yet developed.

Abbreviations: CRS, cherry-red spot; HBOT, hyperbaric oxygen therapy.

time to irreversible anoxic retinal damage and the need for direct evaluation of the damaged tissue. There was a trend (which did not reach statistical significance, $P=0.06$ ) in time delay from symptoms to treatment in the non-CRS group compared to the CRS group.

Due to the great variability between patients, occlusions and residual perfusion, time itself cannot serve as a valid predictor for treatment efficacy. Instead, CRS can serve as a biological marker representing complete anoxia and irreversible infarction of the macular area. Thus, CRS may integrate both time and severity of disease into one parameter. Until CRS develops, irrespective of the time elapsed, reversal of the damage by hyper-oxygenation is possible.

\section{HBOT physiology in CRAO}

Animal models have shown that during a complete clamping of the central retinal artery, there was no correlation between the residual retinal circulation and the retinal damage due to the very low oxygen tension in the blood of residual retinal circulation under normobaric enviornment. ${ }^{5}$ Using 
supplemental oxygen, the choroidal vessels can supply sufficient oxygen to the inner layers of the retina by diffusion, maintaining ganglion cell viability even when the retinal vessels have been completely obliterated. ${ }^{30}$ HBOT incorporates the inhalation of $100 \%$ oxygen at pressures exceeding 1 ATA, thus increasing the amount of oxygen dissolved in the plasma 20-30 times. ${ }^{12}$ Normally, under normoxic conditions, approximately $60 \%$ of the retina's oxygen consumption is supplied from the choroidal circulation, whereas, under such hyperoxic conditions, the choroid is capable of supplying $100 \%$ of the oxygen needed by the retina. ${ }^{31}$

Although elevated partial pressures of oxygen can cause retinal artery vasoconstriction, ${ }^{32,33}$ it does not reduce the oxygen supply when hyperbaric oxygen (via HBOT) is applied. Unlike retinal blood flow, choroidal blood flow is not affected by changes in oxygen tension so that the huge amount of oxygen delivered by HBOT significantly increases retinal oxygenation by diffusion from the unaffected choroid. ${ }^{31,34}$ Oxygenation of the ischemic inner retinal layers by HBOT, under CRAO conditions, is dependent on the choroid perfusion. Therefore, if the level of occlusion is at the ophthalmic artery, the blood supply to the posterior ciliary vessels is blocked as well, and there is no collateral circulation to provide oxygenation to the inner retina..$^{24,35}$

\section{HBOT is effective in ischemic but not necrotic tissue}

According to the current findings, HBOT is effective as long as the ischemic macula has not developed the irreversible anoxic change seen as CRS. HBOT can oxygenate the ischemic, yet not fully necrotic retina, reverse the pre-infarction and significantly improve the visual outcome (86\% clinically significant improvement) (Figure 3). As discussed earlier, the time from ischemia to infarct cannot be fully predicted in humans and depends on many factors. Therefore, although the time delay from symptoms to treatment should be as short as possible, the presence of necrosis/ CRS is an independent predictor for HBOT efficacy.

It should be noted that the same principles are known and have been used for years in the application of HBOT for non-healing ischemic wounds. HBOT can promote healing of ischemic viable tissues via different mechanisms including oxygenation as long as there is no necrosis. ${ }^{36,37}$ The non-viable, necrotic tissue cannot be oxygenated even with HBOT and therefore it is being surgically removed.

\section{CRAO prognosis}

The CRS as a marker of prognosis is of significant importance for evaluation of the risk/cost/benefit per the specific patient in whom HBOT is not easily accessible.
Patients who present with CRS at admission raise regarding the proper management, as HBOT offers limited clinical effect. In the authors' opinion, wherever HBOT is easily available a single sessions should be given and continued only in case of there being a significant improvement during that session.

\section{HBOT safety}

When compared to other means of treatment such as LIF or paracentesis, HBOT is a non-invasive, safe treatment while used for different applications ${ }^{38}$ and was found to be safe also for CRAO patients. The adverse effects, in 5.5\% of the CRAO population, were mild and reversible including middle ear or sinus barotraumas, with complete resolution after several days.

\section{HBOT cost}

HBOT may cost $\$ 1,000-\$ 2,000$ (USD) per session for in hospitals' chambers, which can sum to cost of $\$ 5,000$ $\$ 10,000$ for overall treatment of one CRAO patient. These fees are considered relatively low when compared with other suggested interventions such as the LIF, and when taking into consideration the financial burden related to blindness.

\section{Strengths and limitations}

The current study has several strengths and limitations. Most of the limitations are related to the fact that data were collected retrospectively. There is no control group in the study, and such group would be hard to obtain due to ethical reasons (CRAO is approved indication for HBOT). However, since the visual improvement occurred quite dramatically, within minutes after the target treatment pressure was obtained (while the patient was in the chamber), and since there was not any improvement until HBOT session started, one can conclude that the improvement is related to the increased retinal oxygenation with HBOT. In addition, there was nonconsistent use of different IOP-reduction methods in some of the patients prior to HBOT.

With regard to strengths, a series of 128 patients is the largest cohort reported for CRAO patients treated with HBOT. Strict inclusion and exclusion criteria were used (similar to the EAGLE study ${ }^{11}$ ) in order to carefully examine a defined population of non-arteritic CRAO without a patent cilioretinal artery. Nevertheless, further randomized controlled trials should be performed to evaluate the optimal protocol of HBOT in CRAO patients.

\section{Conclusion}

HBOT is an effective treatment for non-arteritic CRAO as long as CRS, as marker of retinal infarction, has not 
developed. HBOT is a safe, non-invasive intervention that should be considered in CRAO patients. The fundus findings, rather than the time delay from symptoms onset, should be used as the most important marker for treatment success. In future planned protocols, CRS should serve as an important marker for evaluation of any suggested intervention aiming to reverse retinal ischemia.

\section{Acknowledgments}

The authors thank Mr Raz Tuval for reviewing and proofing the manuscript. Study was funded by Research Fund, AssafHarofeh Medical Center, Israel.

\section{Disclosure}

The authors report no conflicts of interest in this work.

\section{References}

1. Rumelt S, Dorenboim Y, Rehany U. Aggressive systematic treatment for central retinal artery occlusion. Am J Ophthalmol. 1999;128(6): 733-738.

2. Augsburger JJ, Magargal LE. Visual prognosis following treatment of acute central retinal artery obstruction. Br J Ophthalmol. 1980;64(12): 913-917.

3. Brown GC, Magargal LE. Central retinal artery obstruction and visual acuity. Ophthalmology. 1982;89(1):14-19.

4. Karjalainen K. Occlusion of the central retinal artery and retinal branch arterioles. A clinical, tonographic and fluorescein angiographic study of 175 patients. Acta Ophthalmol Suppl. 1971;109:1-95.

5. Hayreh SS, Zimmerman MB, Kimura A, Sanon A. Central retinal artery occlusion. Retinal survival time. Exp Eye Res. 2004;78(3):723-736.

6. Landa E, Rehany U, Rumelt S. Visual functions following recovery from non-arteritic central retinal artery occlusion. Ophthalmic Surg Lasers Imaging. 2004;35(2):103-108.

7. Hayreh SS, Zimmerman MB. Central retinal artery occlusion: visual outcome. Am J Ophthalmol. 2005;140(3):376-391.

8. Stone R, Zink H, Klingele T, Burde RM. Visual recovery after central retinal artery occlusion: two cases. Ann Ophthalmol. 1977;9(4):445-450.

9. Duker JS, Brown GC. Recovery following acute obstruction of the retinal and choroidal circulations. A case history. Retina. 1988;8(4):257-260.

10. Neubauer AS, Mueller AJ, Schriever S, Gruterich M, Ulbig M, Kampik A. [Minimally invasive therapy for clinically complete central retinal artery occlusion - results and meta-analysis of literature]. Klinische Monatsblatter fur Augenheilkunde. 2000;217(1):30-36. German.

11. Schumacher M, Schmidt D, Jurklies B, et al. Central retinal artery occlusion: local intra-arterial fibrinolysis versus conservative treatment, a multicenter randomized trial. Ophthalmology. 2010;117(7):1367-1375.

12. Fosen KM, Thom SR. Hyperbaric oxygen, vasculogenic stem cells, and wound healing. Antioxid Redox Signal. 2014;21(11):1634-1647.

13. Weiss JN. Hyperbaric oxygen treatment of nonacute central retinal artery occlusion. Undersea Hyperb Med. 2009;36(6):401-405.

14. Weinberger AW, Siekmann UP, Wolf S, Rossaint R, Kirchhof B, Schrage NF. [Treatment of Acute Central Retinal Artery Occlusion (CRAO) by Hyperbaric Oxygenation Therapy (HBO)-Pilot study with 21 patients]. Klinische Monatsblatter fur Augenheilkunde. 2002; 219(10):728-734. German.

15. Beiran I, Reissman P, Scharf J, Nahum Z, Miller B. Hyperbaric oxygenation combined with nifedipine treatment for recent-onset retinal artery occlusion. Eur J Ophthalmol. 1993;3(2):89-94.

16. Aisenbrey S, Krott R, Heller R, Krauss D, Rossler G, Heimann K. [Hyperbaric oxygen therapy in retinal artery occlusion]. Der Ophthalmologe: Zeitschrift der Deutschen Ophthalmologischen Gesellschaft. 2000;97(7):461-467. German.
17. Menzel-Severing J, Siekmann U, Weinberger A, Roessler G, Walter P, Mazinani B. Early hyperbaric oxygen treatment for nonarteritic central retinal artery obstruction. Am J Ophthalmol. 2012;153(3):454-459.

18. Cope A, Eggert JV, O'Brien E. Retinal artery occlusion: visual outcome after treatment with hyperbaric oxygen. Diving Hyperb Med. 2011;41(3): 135-138.

19. Anderson B Jr, Saltzman HA, Heyman A. The effects of hyperbaric oxygenation on retinal arterial occlusion. Arch Ophthalmol. 1965;73:315-319.

20. Beiran I, Goldenberg I, Adir Y, Tamir A, Shupak A, Miller B. Early hyperbaric oxygen therapy for retinal artery occlusion. Eur $J$ Ophthalmol. 2001;11(4):345-350.

21. Perkins SA, Magargal LE, Augsburger JJ, Sanborn GE. The idling retina: reversible visual loss in central retinal artery obstruction. Ann Ophthalmol. 1987;19(1):3-6.

22. Arditi A, Cagenello R. On the statistical reliability of letter-chart visual acuity measurements. Invest Ophthalmol Vis Sci. 1993;34(1): 120-129.

23. Lange C, Feltgen N, Junker B, Schulze-Bonsel K, Bach M. Resolving the clinical acuity categories "hand motion" and "counting fingers" using the Freiburg Visual Acuity Test (FrACT). Graefes Arch Clin Exp Ophthal. 2009;247(1):137-142.

24. Murphy-Lavoie H, Butler F, Hagan C. Central retinal artery occlusion treated with oxygen: a literature review and treatment algorithm. Undersea Hyperb Med. 2012;39(5):943-953.

25. Snodderly DM, Weinhaus RS, Choi JC. Neural-vascular relationships in central retina of macaque monkeys (Macaca fascicularis). JNeurosci. 1992;12(4):1169-1193.

26. Yu DY, Yu PK, Cringle SJ, Kang MH, Su EN. Functional and morphological characteristics of the retinal and choroidal vasculature. Prog Ret Eye Res. 2014;40:53-93.

27. Suvarna JC, Hajela SA. Cherry-red spot. J Postgrad Med. 2008;54(1): 54-57.

28. Chen H, Chen X, Qiu Z, et al. Quantitative analysis of retinal layers' optical intensities on 3D optical coherence tomography for central retinal artery occlusion. Sci Rep. 2015;5:9269.

29. Osborne NN, Casson RJ, Wood JP, Chidlow G, Graham M, Melena J. Retinal ischemia: mechanisms of damage and potential therapeutic strategies. Prog Ret Eye Res. 2004;23(1):91-147.

30. Patz A. Oxygen inhalation in retinal arterial occlusion; a preliminary report. Am J Ophthalmol. 1955;40(6):789-795.

31. Li HK, Dejean BJ, Tang RA. Reversal of visual loss with hyperbaric oxygen treatment in a patient with Susac syndrome. Ophthalmology. 1996;103(12):2091-2098.

32. Saltzman HA, Hart L, Sieker HO, Duffy EJ. Retinal vascular response to hyperbaric oxygenation. JAMA. 1965;191(4):290-292.

33. Haddad HM, Leopold IH. Effect of hyperbaric oxygenation on microcirculation: use in therapy of retinal vascular disorders. Invest Ophthalmol. 1965;4(6):1141-1151.

34. Yu DY, Cringle SJ. Retinal degeneration and local oxygen metabolism. Exp Eye Res. 2005;80(6):745-751.

35. Mori K, Ohta K, Nagano S, Toshinori M, Yago T, Ichinose Y. [A case of ophthalmic artery obstruction following autologous fat injection in the glabellar area]. Nippon Ganka Gakkai zasshi. 2007;111(1):22-25. Japanese.

36. Efrati S, Bergan J, Fishlev G, Tishler M, Golik A, Gall N. Hyperbaric oxygen therapy for nonhealing vasculitic ulcers. Clin Exp Dermatol. 2007;32(1):12-17.

37. Stoekenbroek RM, Santema TB, Legemate DA, Ubbink DT, van den Brink A, Koelemay MJ. Hyperbaric oxygen for the treatment of diabetic foot ulcers: a systematic review. Eur J Vasc Endovasc Surg. 2014;47(6):647-655.

38. Hadanny A, Meir O, Bechor Y, Fishlev G, Bergan J, Efrati S. The safety of hyperbaric oxygen treatment - retrospective analysis in 2,334 patients. Undersea \& hyperbaric medicine: journal of the Undersea and Hyperbaric Medical Society. 2016;43(2):113-122. 


\section{Supplementary materials}
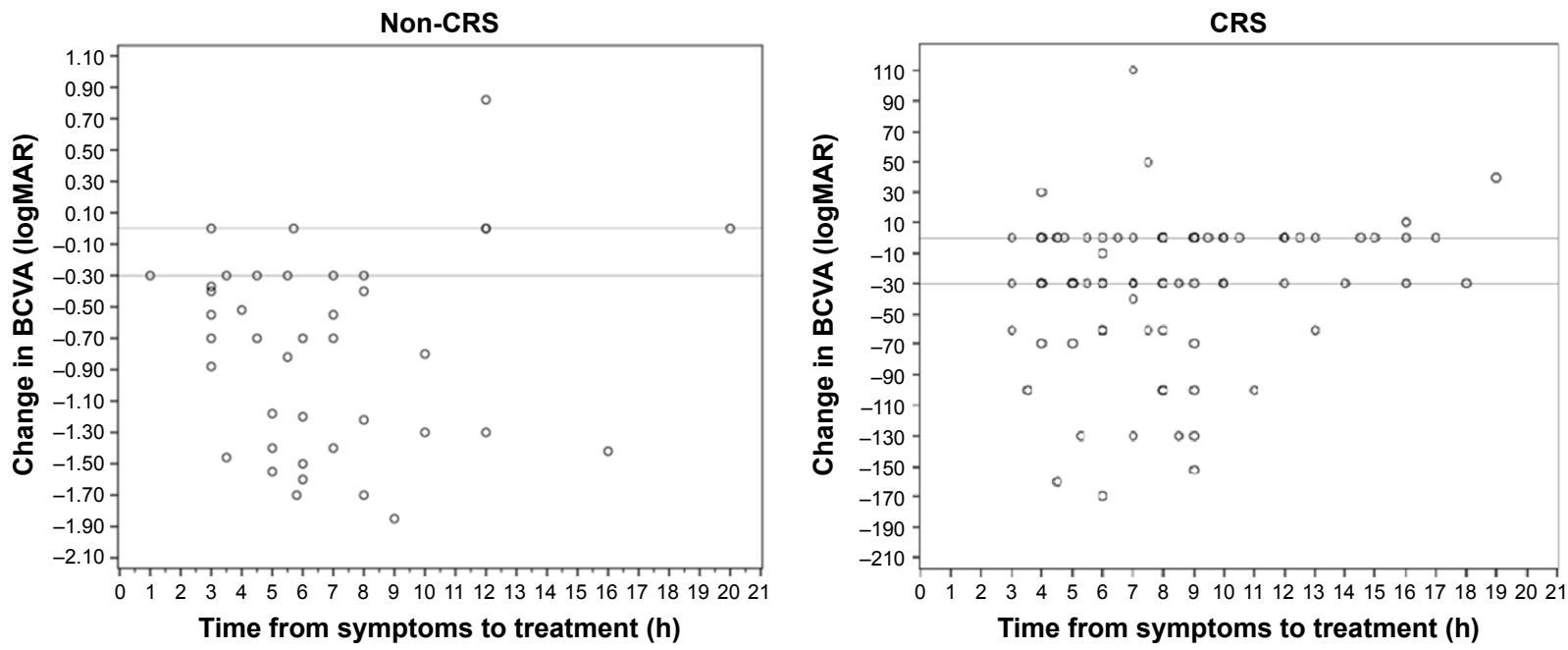

Figure SI The change in BCVA after hyperbaric oxygen therapy as a factor of time delay to treatment.

Abbreviations: CRS, cherry-red spot; logMAR, logarithm of the minimum angle of resolution; BCVA, best-corrected visual acuity.

Table SI Predictors of non-clinically significant improvement $(<0.3 \log M A R)$

\begin{tabular}{|c|c|c|c|c|c|c|}
\hline \multirow[t]{2}{*}{ Variables } & \multicolumn{3}{|c|}{ Univariate } & \multicolumn{3}{|c|}{ Multivariate } \\
\hline & $\overline{\text { OR }}$ & $95 \% \mathrm{Cl}$ & $\overline{\text { Significance }}$ & $\overline{\text { OR }}$ & $95 \% \mathrm{Cl}$ & Significance \\
\hline Baseline logMAR & 0.521 & $0.252-1.074$ & $P=0.077$ & 0.146 & $0.047-0.457$ & $P=0.001$ \\
\hline Hypercholesterolemia & 0.582 & $0.272-1.244$ & $P=0.163$ & $\mathrm{NI}$ & & \\
\hline Stroke & 0.658 & $0.169-2.570$ & $P=0.547$ & $\mathrm{NI}$ & & \\
\hline Right eye & 0.667 & $0.317-1.403$ & $P=0.286$ & $\mathrm{NI}$ & & \\
\hline Number of HBOT sessions & 0.698 & $0.487-1.001$ & $P=0.05 \mathrm{I}$ & $\mathrm{NI}$ & & \\
\hline Active smoking & 0.71 & $0.318-1.58 \mid$ & $P=0.402$ & $\mathrm{NI}$ & & \\
\hline Massage & 0.721 & $0.309-|.68|$ & $P=0.449$ & $\mathrm{NI}$ & & \\
\hline Other vasculopathy & 0.729 & $0.263-2.024$ & $P=0.544$ & $\mathrm{NI}$ & & \\
\hline Previous aspirin & 0.742 & $0.349-1.576$ & $P=0.437$ & $\mathrm{NI}$ & & \\
\hline Ischemic heart disease & 0.775 & $0.340-1.769$ & $P=0.546$ & $\mathrm{NI}$ & & \\
\hline Boxcarring in fundus & 0.83 & $0.330-2.089$ & $P=0.692$ & $\mathrm{NI}$ & & \\
\hline Aspirin & 0.868 & $0.2|3-3.54|$ & $P=0.844$ & $\mathrm{NI}$ & & \\
\hline Hypertension & 0.916 & $0.427-1.964$ & $P=0.821$ & $\mathrm{NI}$ & & \\
\hline IOP & 0.943 & $0.856-1.038$ & $P=0.232$ & $\mathrm{NI}$ & & \\
\hline Acetazolamide PO & 0.962 & $0.393-2.356$ & $P=0.932$ & $\mathrm{NI}$ & & \\
\hline Age & 1.006 & $0.978-1.035$ & $P=0.677$ & $\mathrm{NI}$ & & \\
\hline Diabetes mellitus & 1.03 & $0.418-2.539$ & $P=0.948$ & $\mathrm{NI}$ & & \\
\hline Sex & 1.154 & $0.524-2.54$ I & $P=0.722$ & $\mathrm{NI}$ & & \\
\hline Previous anti-coagulation & 1.188 & $0.328-4.307$ & $P=0.793$ & $\mathrm{NI}$ & & \\
\hline Time delay symptoms to $\mathrm{Tx}$ & 1.208 & I.085-I.345 & $P=0.00 \mathrm{I}$ & $1.25 *$ & I.099-1.422 & $P=0.001$ \\
\hline Previous statin & 1.42 & $0.655-3.080$ & $P=0.375$ & $\mathrm{NI}$ & & \\
\hline Plaque in fundus & 1.451 & $0.543-3.876$ & $P=0.458$ & $\mathrm{NI}$ & & \\
\hline Paracentesis & 1.457 & $0.694-3.056$ & $P=0.320$ & $\mathrm{NI}$ & & \\
\hline Acetazolamide eye & 1.86 & $0.704-4.912$ & $P=0.211$ & $\mathrm{NI}$ & & \\
\hline Cherry-red spot & 4.531 & I.728-II.879 & $P=0.002$ & $|3.15|^{*}$ & $2.964-58.345$ & $P=0.001$ \\
\hline
\end{tabular}

Notes: Baseline BCVA, the time delay from symptoms to treatment, and the fundus finding of CRS at presentation were the only significant variables after multivariate analysis. CRS presence was the most significant predictor with OR of $13.15 \mathrm{I}$ for non-clinically significant improvement. Bold text marks statistical significance $(P<0.05)$, *marks statistical significance in multivariate analysis.

Abbreviations: logMAR, logarithm of the minimum angle of resolution; OR, odds ratio; $\mathrm{Cl}$, confidence interval; $\mathrm{NI}$, not included in multivariate analysis due to nonsignificance; HBOT, hyperbaric oxygen therapy; IOP, intraocular pressure; Tx, treatment; BCVA, best-corrected visual acuity; CRS, cherry-red spot; PO, per oral. 
Table S2 Predictors of bad outcome (BCVA > I logMAR)

\begin{tabular}{|c|c|c|c|c|c|c|}
\hline \multirow[t]{2}{*}{ Variables } & \multicolumn{3}{|c|}{ Univariate } & \multicolumn{3}{|c|}{ Multivariate } \\
\hline & OR & $95 \% \mathrm{Cl}$ & Significance & OR & $95 \% \mathrm{Cl}$ & Significance \\
\hline Right eye & 0.587 & $0.24 \mid-1.432$ & $P=0.24 I$ & $\mathrm{NI}$ & & \\
\hline Hypercholesterolemia & 0.628 & $0.270-1.457$ & $P=0.278$ & $\mathrm{NI}$ & & \\
\hline Hypertension & 0.773 & $0.317-1.882$ & $P=0.570$ & $\mathrm{NI}$ & & \\
\hline Number of HBOT sessions & 0.819 & $0.577-1.1162$ & $P=0.263$ & $\mathrm{NI}$ & & \\
\hline Previous aspirin & 0.836 & $0.360-1.940$ & $P=0.676$ & $\mathrm{NI}$ & & \\
\hline Sex & 0.948 & $0.386-2.331$ & $P=0.908$ & $\mathrm{NI}$ & & \\
\hline Diabetes mellitus & 0.975 & $0.350-2.711$ & $P=0.96 \mathrm{I}$ & $\mathrm{NI}$ & & \\
\hline IOP & 0.989 & $0.902-1.084$ & $P=0.812$ & $\mathrm{NI}$ & & \\
\hline Age & 1.015 & $0.984-1.048$ & $P=0.677$ & $\mathrm{NI}$ & & \\
\hline Previous statin & 1.04 & $0.424-2.547$ & $P=0.932$ & $\mathrm{NI}$ & & \\
\hline Time delay symptoms to $T x$ & 1.125 & $0.985-1.285$ & $P=0.083$ & $\mathrm{NI}$ & & \\
\hline Plaque in fundus & 1.143 & $0.349-3.74 I$ & $P=0.825$ & $\mathrm{NI}$ & & \\
\hline Massage & 1.225 & $0.470-3.195$ & $P=0.678$ & $\mathrm{NI}$ & & \\
\hline Other vasculopathy & 1.317 & $0.407-4.265$ & $P=0.646$ & $\mathrm{NI}$ & & \\
\hline Stroke & 1.444 & $0.298-7.010$ & $P=0.648$ & $\mathrm{NI}$ & & \\
\hline Active smoking & 1.687 & $0.654-4.354$ & $P=0.279$ & $\mathrm{NI}$ & & \\
\hline Ischemic heart disease & 1.725 & $0.638-4.670$ & $P=0.283$ & $\mathrm{NI}$ & & \\
\hline Boxcarring in fundus & 1.792 & $0.564-5.697$ & $P=0.323$ & $\mathrm{NI}$ & & \\
\hline Paracentesis & 2.602 & I.049-6.457 & $P=0.039$ & NI & & \\
\hline Aspirin & 2.67 & $0.324-22.029$ & $P=0.362$ & $\mathrm{NI}$ & & \\
\hline Acetazolamide PO & 2.778 & $0.772-9.993$ & $P=0.118$ & $\mathrm{NI}$ & & \\
\hline Acetazolamide E & 2.854 & $0.620-13.126$ & $P=0.178$ & $\mathrm{NI}$ & & \\
\hline Previous anti-coagulation & 3.000 & $0.367-24.502$ & $P=0.305$ & $\mathrm{NI}$ & & \\
\hline Baseline logMAR & 6.115 & $2.488-15.029$ & $P<0.000$ I & 3.993 & $1.277-12.490$ & $P=0.017$ \\
\hline Cherry-red spot & 25.579 & 7.935-82.455 & $P<0.000$ I & 16.488 & $4.857-55.979$ & $P<0.000$ I \\
\hline \multicolumn{7}{|c|}{$\begin{array}{l}\text { Notes: Baseline BCVA and presence of CRS at presentation were the only significant variables after multivariate analysis. The fundus finding of } C R S \text { at presentation was the } \\
\text { strongest predictor with OR of } 16.488 \text { for a bad outcome. Bold text marks statistical significance }(P<0.05) \text {. } \\
\text { Abbreviations: BCVA, best-corrected visual acuity; logMAR, logarithm of the minimum angle of resolution; OR, odds ratio; Cl, confidence interval; NI, not included in } \\
\text { multivariate analysis due to non-significance; HBOT, hyperbaric oxygen therapy; IOP, intraocular pressure; Tx, treatment; CRS, cherry-red spot; PO, per oral; E, eye drops. }\end{array}$} \\
\hline \multicolumn{7}{|c|}{ Table S3 Analysis of best-corrected visual acuity for patients with time delay from symptoms onset to treatment over 20 hours } \\
\hline Outcome & & $\begin{array}{l}\text { All patients } \\
(n=14)\end{array}$ & $\begin{array}{l}\text { CRS } \\
(n=9)\end{array}$ & & $\begin{array}{l}\text { Non-CRS } \\
(n=5)\end{array}$ & Significance \\
\hline Baseline logMAR & & $2.06 \pm 0.68$ & $2.07 \pm 0.80$ & & $2.0 I \pm 0.20$ & $P=0.874$ \\
\hline Discharge logMAR & & $1.76 \pm 0.94$ & $2.04 \pm 0.75$ & & $0.87 \pm 0.98$ & $P=0.054$ \\
\hline Change in logMAR & & $0.305 \pm 0.678$ & $0.033 \pm 0.234$ & & $1.12 \pm 0.98$ & $P=0.194$ \\
\hline $\begin{array}{l}\text { Clinically significant visual } \\
\text { improvement } \geq 0.3 \text { logMAR }\end{array}$ & & $3(2 \mid \%)$ & I (II.I\%) & & $4(80 \%)$ & $P=0.127$ \\
\hline $\log M A R \leq 1$ & & $4(28 \%)$ & $2(22.2 \%)$ & & $3(60 \%)$ & $P=0.236$ \\
\hline
\end{tabular}

Abbreviations: CRS, cherry-red spot; logMAR, logarithm of the minimum angle of resolution.

Clinical Ophthalmology

\section{Publish your work in this journal}

Clinical Ophthalmology is an international, peer-reviewed journal covering all subspecialties within ophthalmology. Key topics include: Optometry; Visual science; Pharmacology and drug therapy in eye diseases; Basic Sciences; Primary and Secondary eye care; Patien Safety and Quality of Care Improvements. This journal is indexed on

\section{Dovepress}

PubMed Central and CAS, and is the official journal of The Society of Clinical Ophthalmology (SCO). The manuscript management system is completely online and includes a very quick and fair peer-review system, which is all easy to use. Visit http://www.dovepress.com/ testimonials.php to read real quotes from published authors. 